\title{
Microencapsulation methods for plants biologically active compounds - a review
}

\author{
Jelena Mudrić ${ }^{1,}$, , Svetlana Ibrić ${ }^{2}$, AND Jelena Đuriš ${ }^{2}$ \\ ${ }^{1}$ Institute for Medicinal Plants Research "Dr Josif Pančić", Tadeuša Košćuška 1, 11000 Belgrade, Serbia \\ ${ }^{2}$ University of Belgrade, Faculty of Pharmacy, Department of Pharmaceutical Technology and Cosmetology, Vojvode Stepe 450, 11000 Belgrade, Serbia \\ *Corresponding author: jmudric@mocbilja.rs
}

Received: October 29, 2018

Accepted: November 16, 2018

Published on-line: December 20, 2018

Published:December 25, 2018

\begin{abstract}
Biologically active compounds from plants have attracted great interest due to their affordability, effectiveness and low toxicity. Herbal extracts provide an infinite resource of raw materials for pharmaceutical, cosmetic and food industry. Unfortunately, use of the valuable natural compounds can be limited by their low bioavailability, volatilization of active compounds, sensitivity to the temperature, oxidation and UV light, in vivo instability, as well as unpleasant taste. One of the potential strategies to overcome these issues is microencapsulation of the biologically active ingredients. In this review, preparation, applications and limitations of the most popular techniques for microencapsulation, such as spray drying, fluid bed coating, encapsulation using supercritical fluids, freeze drying, ionic gelation, emulsification-solvent removal methods and formulation of liposomes, were discussed. Also, microparticles properties produced by presented microencapsulation methods were interpreted.
\end{abstract}

Key words: herbal extracts; medicinal plants; microencapsulation; polyphenols; microparticles; antioxidants

http://dx.doi.org/10.5937/leksir1838062M

\section{INTRODUCTION}

According to the available data, nowdays $80 \%$ of the world's population uses herbs and herbal medicaments for treatment of various ailments. The acceptability of herbal formulations is increasing due to their affordability, effectiveness and low toxicity (Atul Bhattaram et al., 2002). Unfortunately, use of the valuable natural compounds can be limited by their unpleasant taste, low bioavailability, volatilization of active compounds, sensitivity to the temperature, oxidation and UV light, as well as in vivo instability (Ćujić et al., 2013; Abd Manaf et al., 2015; Munin and Edwards-Levy, 2011). One of the potential strategies to overcome these issues is microencapsulation of the herbal active ingredients. Therefore, microencapsulation technology can be used for obtaining herbal products with desirable characteristics. Microencapsulation techniques of plant materials and other natural products are widely used in the food, pharmaceutical and cosmetic industries.

Microencapsulation is a process by which solids, liquids or gases are surrounded with a membrane or matrix (Desai and Jin Park, 2005; Suri et al., 2013). The entrapped material is known as the core material, actives, fill, internal phase or payload. The coating material can be called the capsule, wall material, membrane, carrier or shell (Gibbs et al., 1999). The particles with sizes ranging from 1 to $1000 \mu \mathrm{m}$ are called microparticles or microspheres. There are two types of microparticles; microcapsules and micromatrices (Figure 1). Microcapsules are made of one or multiple core substances,surrounded by a distinct capsule wall. Micromatrices are polymeric matrices in which the encapsulated substances are homogeneously dispersed (Park and Yeo, 2007). Terminology in this field is inconsistent, sometimes micromatrices are also called microspheres. Depending of the entrapped materials properties, core materials and methods used for preparation, particles with different structure, size and shape can be obtained (Poshadri and Aparna, 2010).

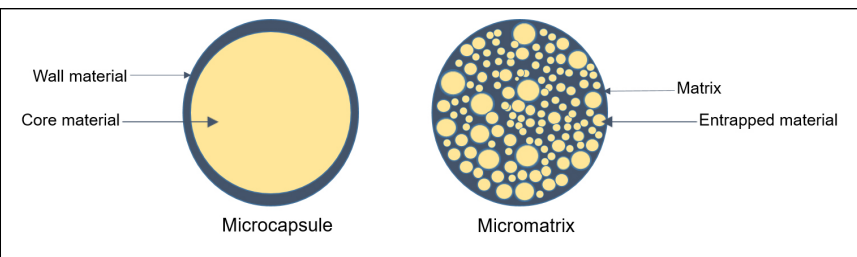

Fig. 1. Two major types of microparticles: microcapsules and micromatices

Microencapsulation can be used to enhance different biologically active compounds properties (Desai and Jin Park, 2005; Singh et al., 2010):

- Improve the stability of the encapsulated bioactive compounds and to extend their shelf life

- Reduce their degradation 
- Enhance physical performance and promote easier handling

- Mask the unpleasant taste of substances

- Prevent interactions

- Enable modified release

- Reduce toxicity

- Improve efficiency and/or compliance (Figure 2)

In order to obtain the products of the desired characteristics, it is necessary to consider: expected effect of the encapsulated bioactive substance(s), preferable particle size, density, stability requirements for the encapsulated ingredient(s), optimal concentration of the active principle(s) in microparticles, way of release and delivery of bioactive molecule(s), coating material properties, processing conditions that are expected to be used for preparation of the microparticle and the cost limits (Desai and Jin Park, 2005).

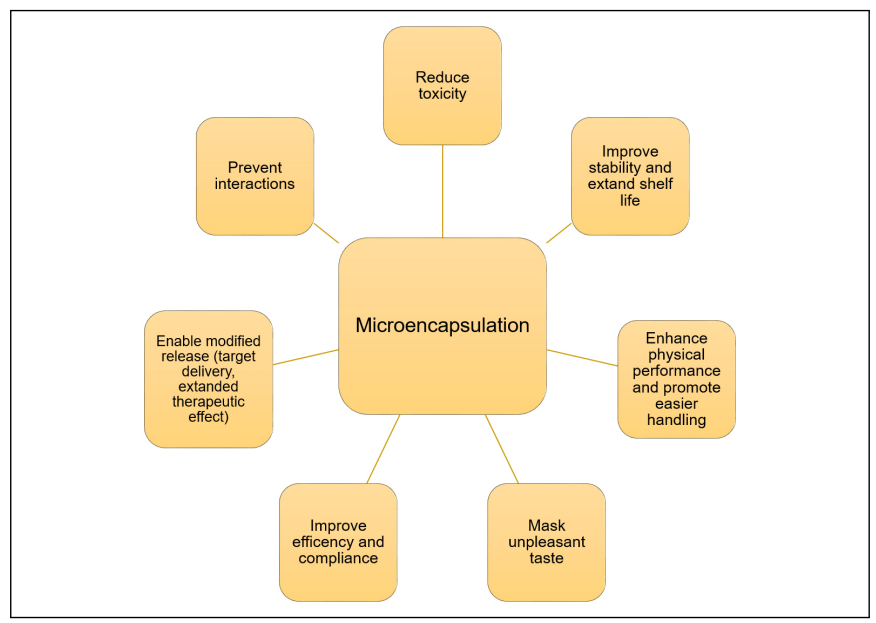

Fig. 2. Application of microencapsulation in food and pharmaceutical industry

\section{MICROENCAPSULATION METHODS}

Microencapsulated particles can be prepared by several microencapsulation techniques. The choice of the microencapsulation process is governed by the physical and chemical properties of the core and coating materials and the intended application. Various encapsulation methods have been previously used for encapsulation of plant or natural products. Some of the most popular techniques, such as spray drying, fluid bed coating, encapsulation using supercritical fluids, freeze drying, ionic gelation, emulsification-solvent removal methods and formulation of liposomes are presented in this review.

\subsection{Spray drying}

Spray drying is a widely applied method used in the food, chemical, electronics, pharmaceutical and biopharmaceutical industry (Jain et al., 2012). In the pharmaceutical industry, this technique could be applied in case of microencapsulation, granulation and complex formation. Also, spray drying is successfully used for the modification of biopharmaceutical properties of drugs and the formulation of dry powder aerosols and heat sensitive materials. This method is often used to encapsulate drugs, plant extracts, essential oils and various biologically active compounds (Belščak-Cvitanović et al., 2015; Ćujić, 2017; Dias et al., 2017; Peres et al., 2011).

The principle of microencapsulation by spray drying is based on the dissolution or dispersion of the core material in the carrier solution, dispersed in the hot air stream. The rapid evaporation of solvent causes the entrapment of the active compound into the carrier (Cocero et al., 2009). The solvent (dispersing agent) quickly evaporates, while the incorporated substance is briefly exposed to the hot air stream. The particles are collected after they fall on the bottom of the dryer (Snyder and Lechuga-Ballesteros, 2008). Numerous properties of particles can be optimized (particles shape and size, porosity, bulk density, flow rate, moisture content, etc.) by choosing the appropriate materials, equipment and process parameters. The advantages of spray drying: the ability to obtain the high quality product and to control its properties, the stability of the obtained product is improved, scale-up is simple and production costs are relatively low (Miletic, 2013). During the spray drying process of plant extracts with high sugar content, adhesion to the walls may occur as a problem, due to the low temperature of the glass transition of sugars and organic acids. This problem can be overcome using the carriers with high glass transition temperature, such as gum arabic, maltodextrins, starches, proteins, hydrocolloids and various hydrolysed starches (Tomšik, 2018).

It has been reported that the antioxidant capacity of Quercus resinosa leaves infusions was high. On the other hand, Quercus resinosa leaves are well known as adstrigens. This plant material is also unstable due to polyphenolic compounds and interactions with different food ingredients. For this reason, the aim of the work by Rocha-Guzman et al. (2010) was to obtain encapsulated Quercus resinosa leaves infusions with appropriate stability and masked taste by the spray drying method. Sodium caseinate and lactose monohydrate were selected as carriers. Dispersion of lyophilized Quercus resinosa leaves infusions and wall material were homogenized at 0 , 100,200 , and $300 \mathrm{MPa}$. Wall matrix was made of lactose and sodium caseinate blends in 3 ratios (110:40, 90:60, and 70:80 $\mathrm{g} / \mathrm{L})$. Homogenized dispersions were encapsulated by spray drying method. The encapsulated particles of the Quercus resinosa leaves infusions were in the size range from $138.1 \pm 1.1$ to $269.8 \pm 2.9 \mathrm{~nm}$, with significant antioxidant activity.

\subsection{Fluid bed coating}

The fluid bed encapsulation process consists spraying of the coating solution into a fluidized solid particles and several cycles of wetting and drying (Guignon et al., 2002). The coating material forms film over the particle surfaces. When the particles are fluidized, separation force is exerted to the particles, which are more or less agglomerated by the spray solution. Fluid bed processor can generate its inherent separation force, depending on the operating conditions such as the airflow pattern and the inlet airflow rate. In practice, depending on the requirement of the final particle size, the optimal processing conditions should be selected (Park and Yeo, 2007). Coating material must be thermally stable and it is important to have acceptable viscosity to enable pumping and atomizing. Suitable material for encapsulation by this process should be spherical and dense with a narrow particle size distribution and good flowability (Zuidam and Shimoni, 2010). The advantage of this procedure is that the solution of the coating agent can be applied in several layers. Also, there is a possibility of coating with several different materials during the production process. However, this method has some disadvantages and limits. It is difficult to produce particles smaller than $20 \mu \mathrm{m}$. Also, this technique requires large amount of coating materials (Guignon et al., 2002).

Artificial colorants are often used to improve the sensory properties of food and pharmaceutical products. However, using has become controversial because of their toxicological potential. $\beta$-carotene is well-known antioxidant, which can also be used as a colorant, due to its distinct red-yellow color. On 
the other hand, $\beta$-carotene molecules are susceptible to oxidation and thermal degradation. For this reason, the objective of the study published by Coronel-Aguilera and San MartinGonzalez (2015) was to encapsulate the spray dried emulsion of $\beta$-carotene by fluidized bed coating technology and to evaluate the influence of the fluidized bed coating process conditions: the inlet air temperature $\left(60,70\right.$ or $\left.80^{\circ} \mathrm{C}\right)$ and the coating solution feed rate $(7$ or $10 \mathrm{~mL} / \mathrm{min})$. Encapsulation was performed in a fluidizing system with a Wurster column. Coating material consisted of a $10 \%$ solution of hydroxypropyl cellulose. The particle size was in the range from 48.6 to $69.5 \mu \mathrm{m}$. Smaller particle size was obtained with the higher temperature and feeding rate. The greatest encapsulation of $\beta$-carotene was achieved in the case of the lowest temperature and lowest feed rate. This result can be partly explained by the fact that the high temperature may contribute to $\beta$-carotene degradation. The stability of the obtained particles was studied at 4, 25 and $45{ }^{\circ} \mathrm{C}$ during 4 weeks. The temperature showed an effect on the stability of the tested particles. The loss in $\beta$-carotene content was larger at higher temperatures, in comparison to lower temperature. The major degradation in this research was observed during the first week.

\subsection{Encapsulation using supercritical fluids}

Among the numerous conventional encapsulation processes, those based on supercritical technologies have attracted the food and pharmaceutical applications because of the possibility to work with thermolabile products and the ability to engineer the particle (Keven Silva and Angela A. Meireles, 2014).

Supercritical fluids (SCFs) are fluids on the temperature and pressure above the critical pressure and temperature values for the given fluid. This state is often described as a state between the liquid and gas. SCFs have a density similar to liquid, compressibility and viscosity as a gases, whereas the diffusion capacity is higher than that of a liquid (Medarević et al., 2013). Supercritical fluids characteristics can be affected by changing the pressure and temperature of the given system. Supercritical carbon dioxide $\left(\mathrm{scCO}_{2}\right)$ is the supercritical fluid, widely used since it is a non-toxic, chemically inert gas, without secondary environmental products, making it environmentally friendly and GRAS (GRAS-Generally Recognized as Safe). $\mathrm{scCO}_{2}$ has other advantages, such as availablity and affordablity. The supercritical region of $\mathrm{scCO}_{2}$ can be achieved at moderate pressures and temperatures $(\mathrm{Tc}=304.2 \mathrm{~K}, \mathrm{Pc}=$ $7.38 \mathrm{MPa}$ ) making it possible to carry out the process at nearambient temperatures, avoiding the degradation of thermolabile substances (Cocero et al., 2009). Many different encapsulation processes are based on the use of supercritical fluids, in which SCFs performs different functions: acts as a solvent (Rapid Expansion of Supercritical Solutions, RESS), antisolvent (Supercritical Anti Solvent precipitation, SAS), co-solvent or solute (Particles from Gas Saturated Solutions, PGSS), propellant (Carbon dioxide-Assisted Nebulisation with a Bubbler Dryer CAN-BD), etc. (Martin et al., 2010). The use of $\mathrm{scCO}_{2}$ as the antisolvent has been explored for different materials, such as polymers, pharmaceuticals, inorganic compounds, food and plants bioactives (Keven Silva and Angela A. Meireles, 2014; Sosa et al., 2011).

Catechins present in green tea (Camellia sinensis) have low bioavailability (2-5\%) and extensive clearance. Therefore, a group of authors (Sosa et al., 2011) encapsulated green tea polyphenols using a method based on the use of supercritical fluid, which was acting as an anti-solvent. Acetone was used to dissolve green tea extract and carrier, biodegradable polymer (poly- $\epsilon$-caprolactone, MW: 25,000). During this study, the following process parameters influence was examined: the operating pressure (8-12 MPa) and temperature (283-307 K), the polymer to solutes concentration $(\mathrm{w} / \mathrm{w})$ ratio $(4-58)$, and the $\mathrm{CO}_{2}$ to solution mass flow rate ratio (4-10). The particles obtained at a temperature below $290 \mathrm{~K}$ were small (about $5 \pm 2$ $\mu \mathrm{m})$, with high polyphenol content, which means that the efficiency of the encapsulation was high (60-100\%). Polyphenols dissolution rate from microparticles shows that $30 \%$ of the antioxidants were released rapidly for the first 4 hours, which was explained by diffusion mechanism, whereas the largest amount of polyphenols were retained within the crystalline domains of the polymer matrix and was released gradually over the next 90 hours, due to the matrix degradation.

\subsection{Freeze drying}

Freeze drying or lyophilization, is a drying process in which the solvent or the suspension medium, is frozen and then sublimed from the solid state directly into the gaseous phase (Fang and Bhandari, 2012). Prior to drying, active ingredients and polymers are dissolved or dispersed in the medium. The freeze drying process is divided into three basic phases: freezing, primary drying and final (secondary) drying. This technique is simple and suitable for encapsulation of thermosensitive or unstable substances. Also, the moisture content of the final product is controllable, and the dried products generally have appropriate stability and high quality. They can be easily reconstituted in water, because they have a high specific surface area and porous structure. The long duration of the drying phase and the high cost of the process, are the limitations of this method (Rey and May, 2004; Tang and Pikal, 2004; Fang and Bhandari, 2012).

The properties of the freeze-dried, encapsulated products could be affected by the operating conditions and wall material properties. According to the available literature, most of these parameters were chosen based on the trial and error approach (Fang and Bhandari, 2012). Also, the freeze drying process can be used after the other encapsulation methods, in order to convert the encapsulated core materials into the powder form (Stojanovic et al., 2012; Ćujić et al., 2016).

Micropartices lyophilization was used in order to improve the stability of Rubus chamaemorus extract (Laine et al., 2008). It has been shown that freeze drying process improved the extract stability, rich with extremely unstable ellagitannins.

\subsection{Complex coacervation}

The term complex coacervation was introduced by the Dutch chemist Bungenberg de Jong in 1949. The first patented microencapsulation process was based on the phenomenon of complex coacervation (Lakkis, 2016). This method is based on interactions between water-soluble cationic and anionic polymers, which form a polymer-rich phase called coacervate (Munin and Edwards-Levy, 2011). Polymers coacervate could be formed around the dispersed core particles. The formation of coacervate as a special phase is a main precondition for this microencapsulation process. Coacervate can be formed spontaneously by mixing the cationic and anionic polymers or by the addition of an anti-solvent, electrolyte, temperature reduction, adjustment of the medium $\mathrm{pH}$, as well as in the presence of certain surfactants (Sovilj et al., 2000). The charges must be large enough to produce coacervate, but not too large in order to avoid precipitation (Munin and Edwards-Levy, 2011).

Due to anti-inflammatory, antioxidant and antimicrobial properties propolis could be applied in various products, such as toothpaste, shampoos, creams, etc. However, its using is limited due to the solubility only in alcohol, and it has specific taste and odor. The aim of the study by Nori et al. (2011) was to encapsulate the propolis extract by a complex coacervation with soy protein and pectin, without alcohol. The influence of different $\mathrm{pH}$ values (3.5; 4.0; 4.5 and 5) and the influence 
of propolis concentrations $(2.5 \mathrm{~g} / 100 \mathrm{~mL}$ and $5 \mathrm{~g} / 100 \mathrm{~mL})$, as well as pectin $(2.5 \mathrm{~g} / 100 \mathrm{~mL}$ and $5 \mathrm{~g} / 100 \mathrm{~mL})$ and soy protein concentrations $(2.5 \mathrm{~g} / 100 \mathrm{~mL}$ and $5 \mathrm{~g} / 100 \mathrm{~mL})$ were examined. The size of the obtained microcapsules in this research was in the range from 10 to $90 \mu \mathrm{m}$, which was expected for microcapsules produced by complex coacervation. According to the literature, particles size produced by complex coacervation ranged from 1 to $500 \mu \mathrm{m}$. The encapsulation efficiency of formulation using $2.5 \mathrm{~g} / 100 \mathrm{~mL}$ concentration of encapsulants and core was $72.01 \pm 0.01 \%$; whereas microparticles whose encapsulants and core concentration were $5 \mathrm{~g} / 100$ $\mathrm{mL}$ had slightly lower encapsulation efficiency $(66.12 \pm 0.01 \%)$. This result was not expected since increase in the concentration of colloids and the core in solutions was supposed to promote coacervation and provide higher encapsulation efficiency. In this study, it was also shown that the application of coacervation was not deleterious for antioxidant compounds. Furthermore, the antimicrobial activity of the incorporated extract was preserved. The stability of the microcapsules during the 180 days period at 10 and $25^{\circ} \mathrm{C}$ was examined. The conclusion was that these microparticles should be stored at a temperature of $10{ }^{\circ} \mathrm{C}$ in order to preserve the stability of flavonoids.

\subsection{Ionic gelation}

Ionic gelation is a physiochemical microencapsulation technique, which includes cross-linking of polyelectrolytes (or charged polymers) in the presence of multivalent counter ions (Park and Yeo, 2007). This process consists of extruding an aqueous solution of polymer through a syringe needle or a nozzle, in which the core material is dissolved or dispersed. Droplets are received in a dispersant phase and transformed into spherical gel particles, after a reaction of polyelectrolytes and multivalent counter ions (Munin and Edwards-Levy, 2011).

Catechins are a class of polyphenolic flavonoids with confirmed antioxidant activities and diverse biological properties, such as anti-inflammatory, vasodilatory, neuroprotective and chemopreventive effects. On the other hand, catechins are highly unstable. Various factors influence the stability of catechins. However, $\mathrm{pH}$ is the most crucial factor and the rate of oxidation increases as the $\mathrm{pH}$ increases. Therefore, a group of researchers encapsulated catechins with an ion-based gel process (Dube et al., 2010). Catechin and epigallocatin are incorporated into chitosan-tripolyphosphate nanoparticles. These nanoparticles with catechin had an average size of $163 \pm 2 \mathrm{~nm}$, with a polydispersity index of $0.43 \pm 0.02$ and a zeta potential of $33 \pm 1 \mathrm{mV}$. In the case of encapsulated epigallocatechin gallate, the particle size was $165 \pm 2 \mathrm{~nm}$, the polydispersity index was $0.46 \pm 0.2 \mathrm{~nm}$, whereas the zeta potential was $33 \pm 1 \mathrm{mV}$. In general, the nanoparticles in this research had a mean size less than $200 \mathrm{~nm}$ and had a positive zeta potential exceeding $30 \mathrm{mV}$. These attributes are favorable for nanoparticles for oral delivery, since it has been shown that the potential way of uptake of particles having a mean size below $500 \mathrm{~nm}$ is translocation through the gastrointestinal mucous barrier and interactions with the absorptive epithelial cells (Florence and Hussain, 2001). It is stated that the zeta potential of these particles reflects the aspect of stability and inhibition of particle aggregation. Also, the high positive zeta potential may enhance their bioadhesive properties, which may be mediated through interactions with negatively charged cell surfaces. The study also showed that it takes 8 hours to reduce the concentration of the catechin by $50 \%$ in phosphate buffer ( $\mathrm{pH} 7.4)$, whereas for encapsulated catechin $50 \%$ of the initial concentration degraded after 24 hours. For epigallocatechin gallate, the corresponding values were 10 and 40 minutes, respectively.

\subsection{Emulsification-Solvent Removal Methods}

Microparticles can be produced from an emulsion of two or more immiscible liquids. The most commonly used methods are solvent evaporation and the solvent extraction technique. In the solvent evaporation method, a volatile solvent has a very low miscibility with water. This technique is based on the evaporation of the hydrophobic solvent from the emulsion in which microspheres are formed and the active ingredients are trapped in the polymer matrix and encapsulated on that way. First step includes dissolving of the polymer (coating material) in the organic solvent. After that, the active principles are dissolved/dispersed in the obtained polymeric solution. The mixture is emulsified in a large amount of water containing surfactants, to obtain an oil-in-water $(\mathrm{O} / \mathrm{W})$ emulsion. Evaporation of the solvent is achieved by heating under vacuum and with gentle stirring. This method is not applicable in the case of volatile compounds encapsulation and in the case of compounds with high affinity for the continuous phase ( $\mathrm{Lu}$ et al., 2016; Munin and Edwards-Levy, 2011).

The solvent extraction technique, also called nanoprecipitation, is a method where solvent is miscible with water in all proportions. The method involves dissolving/dispersing the bioactive compounds in a solvent in which the polymer has previously been dissolved. Then, this phase is injected under agitation into a continuous aqueous phase containing a surfactant. Nanoparticles are formed by spontaneous diffusion of solvents into the aqueous phase, while a polymer precipitates and forms particles with encapsulated active principles ( $\mathrm{Lu}$ et al., 2016; Munin and Edwards-Levy, 2011). Following the solvent removal, the resulting particles are purified, filtered or centrifuged. Subsequently, they are dried by a conventional method or by lyophilization (Munin and Edwards-Levy, 2011) Resveratrol is a natural polyphenolic compound and it has been reported that resveratrol induces many cellular responses, including cell cycle arrest, differentiation and apoptosis, making it a novel anticancer agent. In the study reported by Shao et al. (2009), the activity of resveratrol in the free form and encapsulated resveratrol was compared, for the treatment of invasive brain tumor (malignant gliomas). mPEGPCL (methoxy poly (ethylene glycol)-poly(caprolactone)), was used as a carrier having a hydrophilic and hydrophobic region. On this way, it is possible to incorporate the hydrophobic compound, such as resveratrol, into the core, which is surrounded by the hydrophilic chains. Resveratrol nanoparticles were prepared by nanoprecipitation method. The particles obtained in this study were spherical with smooth surface, the size of particles was not significantly changed after lyophilization $(78.3 \pm 7.9 \mathrm{~nm}$ prior to lyophilization and $87.5 \pm 9.59 \mathrm{~nm}$ afterwards). The zeta potential prior to drying was $-6.5 \pm 1.4$; whereas afterwards it was $-5.6 \pm 0.7$. The efficiency of encapsulation was higher than $90 \%$. The resveratrol loading content was $19.4 \pm 2.4 \%$. In vitro release of resveratrol loaded in nanoparticles indicated that $50 \%$ of resveratrol was released during the first 5 hours, followed by the observed trend of prolonged release. In vitro study revealed that higher efficiency of encapsulated resveratrol can be explained by higher ability of encapsulated resveratrol penetration in cell membranes in comparison to the same dose of free resveratrol.

\subsection{Liposomes}

Liposomes were discovered around 1965 when British hematologist Dr Bangham described the appearance of liquid crystals of lecithin in contact with water forming structures very similar to biological membranes. Liposomes are mainly composed of phospholipids and due to their amphipathic nature they have a strong tendency to form membranes (Bozzuto and Molinari, 2015). In addition, they are biodegradable and biocompatible vesicular systems. Liposomes could be made up 
of one or more lipid bilayers, which surround the core filled with water. Based on the number of double layers vesicles liposomes can be classified as: multilamellar vesicles (MLVs), oligolamellar vesicles and unilamellar vesicles (ULVs). The specific structure of these carriers makes them suitable for encapsulating compounds of various physicochemical properties. Hydrophilic active substances could be incorporated into the hydrophilic interior, while lipophilic and amphiphilic compounds could be incorporated into the liposomal lipid bilayer (Swami et al., 2015). Large aqueous core represents interior of ULVs, making them suitable for the encapsulation of hydrophilic molecules. On the other hand, MLVs preferably entrap lipid-soluble substances (Bozzuto and Molinari, 2015). Liposome preparation techniques may be divided into 1) bulk methods, whereby liposomes are obtained through transfer of phospholipids from an organic phase into an aqueous phase and 2) film methods, in which lipid films are first deposited on a substrate and then hydrated to give liposomes. Following the preparation of liposomes, various methods for particle size reduction could be used, such as: sonication, extrusion, freeze-thaw cycles or homogenization (Patil and Jadhav, 2014) Amarogentin, secoiridoid glycoside, can be potentially used as antileishmanial agent. Therefore, group of authors evaluated amoragentin efficacy in the free form and in two vesicular forms, liposomes and niosomes (Medda et al., 1999). The amarogentin in both vesicular forms was found to be a more effective antileishmanial agent compared to the free form. Experiments on hamsters were performed with the equivalent drug concentration for free, liposomal and niosomal form of this active principe. Free amarogentin reduced the parasite load by $34 \%$. Liposomal form reduced the parasite load by $69 \%$, whereas niosomal form reduced the parasite load by 90

\section{CONCLUSION}

Plants contain large number of compounds of various physicochemical, biopharmaceutical, pharmacokinetic and pharmacodynamic properties. For this reason, formulation of the herbal products with appropriate properties in terms of efficiency, stability and quality is a complex task. Encapsulation methods can play a key role in achieving these goals. Review of the various research results reveals that encapsulation is a method for protection of natural compounds, increasing their shelf life. It was shown that encapsulation enables controlled release and improves bioavailability and efficiency. Moreover, in some instances encapsulation methods are being used to mask an unpleasant taste. Microencapsulation technique should be selected depending on the desirable characteristics of microparticles (size, entrapment efficacy, drug loading, bioavailability, stability). Although there are plenty possibilities how to encapsulate a biologically active compound, usually two or more encapsulation processing steps are required to get microparticles with preferable characteristics.

\section{ACKNOWLEDGMENTS}

Acknowledgment. This work was supported by the project No. TR 34007, funded by the Ministry of Education, Science and Technological Development, Republic of Serbia.

\section{REFERENCES}

Abd Manaf, M., Jai, J., Raslan, R., Subuki, I. and Mustapa, A. N. (2015). Microencapsulation Methods of Volatile Essential Oils - A Review, Advanced Materials Research 1113: 679-683.

Atul Bhattaram, V., Graefe, U., Kohlert, C., Veit, M. and Derendorf, H. (2002). Pharmacokinetics and Bioavailability of Herbal Medicinal Products, Phytomedicine 9: 1-33.
Belščak-Cvitanović, A., Lević, S., Kalušević, A., Špoljarić, I., Đorđević, V., Komes, D., Mršić, G. and Nedović, V. (2015). Efficiency Assessment of Natural Biopolymers as Encapsulants of Green Tea (Camellia sinensis L.) Bioactive Compounds by Spray Drying, Food and Bioprocess Technology 8(12): 2444-2460.

Bozzuto, G. and Molinari, A. (2015). Liposomes as nanomedical devices, International Journal of Nanomedicine 10: 975-999.

Cocero, M. J., Martin, A., Mattea, F. and Varona, S. (2009). Encapsulation and co-precipitation processes with supercritical fluids: Fundamentals and applications, The Journal of Supercritical Fluids 47(3): 546-555.

Coronel-Aguilera, C. P. and San Martin-Gonzalez, M. F. (2015). Encapsulation of spray dried $\beta$-carotene emulsion by fluidized bed coating technology, LWT - Food Science and Technology 62(1): 187-193.

Ćujić, N. (2017). Optimization of chokeberry (Aronia melanocarpa (Michx.) Elliott) extraction, microencapsulation of extract by electrostatic extrusion and spray drying methods. [Dissertation overview], Lekovite Sirovine 37: 5963.

Ćujić, N., Kundaković, T. and Šavikin, K. (2013). Anthocyanins - chemistry and biological activity, Lekovite Sirovine 33: 1937.

Ćujić, N., Trifković, K., Bugarski, B., Ibrić, S., Pljevljakušić, D. and Šavikin, K. (2016). Chokeberry (Aronia melanocarpa L.) extract loaded in alginate and alginate/inulin system, Industrial Crops and Products 86: 120-131.

Desai, K. G. H. and Jin Park, H. (2005). Recent Developments in Microencapsulation of Food Ingredients, Drying Technology 23(7): 1361-1394.

Dias, D. R., Botrel, D. A., Fernandes, R. V. D. B. and Borges, S. V. (2017). Encapsulation as a tool for bioprocessing of functional foods, Current Opinion in Food Science 13: 31-37.

Dube, A., Ng, K., Nicolazzo, J. A. and Larson, I. (2010). Effective use of reducing agents and nanoparticle encapsulation in stabilizing catechins in alkaline solution, Food Chemistry 122(3): 662-667.

Fang, Z. and Bhandari, B. (2012). Encapsulation technologies and delivery systems for food ingredients and nutraceuticals, in N. Garti and J. McClements (eds), Encapsulation Technologies and Delivery Systems for Food Ingredients and $\mathrm{Nu}$ traceuticals, Elsevier, chapter Spray drying, freeze drying and related processes for food ingredient and nutraceutical encapsulation, pp. 73-109.

Florence, A. T. and Hussain, N. (2001). Transcytosis of nanoparticle and dendrimer delivery systems: evolving vistas, $A d-$ vanced Drug Delivery Reviews 50: S69-S89.

Gibbs, B. F., Kermasha, S., Alli, I. and Mulligan, C. N. (1999). Encapsulation in the food industry: a review, International Journal of Food Sciences and Nutrition 50(3): 213-224.

Guignon, B., Duquenoy, A. and Dumoulin, E. D. (2002). Fluid bed encapsulation of particles: principles and practice, Drying Technology 20(2): 419-447.

Jain, M., Ganesh, L., Manoj, B., Randhir, C., Shashikant, B. and Chirag, S. (2012). Spray Drying in Pharmaceutical Industry: A Review, Research Journal of Pharmaceutical Dosage Forms and Technology 4(2): 74-79. 
Keven Silva, E. and Angela A. Meireles, M. (2014). Encapsulation of Food Compounds Using Supercritical Technologies: Applications of Supercritical Carbon Dioxide as an Antisolvent, Food and Public Health 4(5): 247-258.

Laine, P., Kylli, P., Heinonen, M. and Jouppila, K. (2008). Storage Stability of Microencapsulated Cloudberry (Rubus chamaemorus) Phenolics, Journal of Agricultural and Food Chemistry 56(23): 11251-11261.

Lakkis, J. M. (ed.) (2016). Encapsulation and Controlled Release Technologies in Food Systems: Lakkis/Encapsulation and Controlled Release Technologies in Food Systems, John Wiley \& Sons, Ltd, Chichester, UK.

Lu, W., Kelly, A. L. and Miao, S. (2016). Emulsion-based encapsulation and delivery systems for polyphenols, Trends in Food Science \& Technology 47: 1-9.

Martin, A., Varona, S., Navarrete, A. and Cocero, M. J. (2010). Encapsulation and Co-Precipitation Processes with Supercritical Fluids: Applications with Essential Oils, The Open Chemical Engineering Journal 4(2): 31-41.

Medarević, D., Ibrić, S., Đuriš, J. and Đurić, Z. (2013). Solid dispersion application in pharmaceutical technology: Methods of preparation and characterization, Arhiv za farmaciju 63: 473-493.

Medda, S., Mukhopadhyay, S. and Basu, M. K. (1999). Evaluation of the in-vivo activity and toxicity of amarogentin, an antileishmanial agent, in both liposomal and niosomal forms, Journal of Antimicrobial Chemotherapy 44(6): 791-794.

Miletic, T. M. (2013). Spray drying process characterisation and modelling in the solid dosage forms development by application of quality by design concept, PhD thesis, University of Belgrade, Facylty of Pharmacy, Serbia.

Munin, A. and Edwards-Levy, F. (2011). Encapsulation of Natural Polyphenolic Compounds; a Review, Pharmaceutics 3(4): 793-829.

Nori, M. P., Favaro-Trindade, C. S., Matias de Alencar, S., Thomazini, M., de Camargo Balieiro, J. C. and Contreras Castillo, C. J. (2011). Microencapsulation of propolis extract by complex coacervation, LWT - Food Science and Technology 44(2): 429-435.

Park, K. and Yeo, Y. (2007). Microencapsulation technology, in J. Swarbrick (ed.), Encyclopedia of Pharmaceutical Technology, 3rd edn, Informa Healthcare, New York, chapter Microencapsulation Technology, pp. 2315-2327.

Patil, Y. P. and Jadhav, S. (2014). Novel methods for liposome preparation, Chemistry and Physics of Lipids 177: 8-18.

Peres, I., Rocha, S., Gomes, J., Morais, S., Pereira, M. C. and Coelho, M. (2011). Preservation of catechin antioxidant properties loaded in carbohydrate nanoparticles, Carbohydrate Polymers 86(1): 147-153.

Poshadri, A. and Aparna, K. (2010). Microencapsulation technology: A review, Journal of Research ANGRAU 38: 86-102.

Rey, L. and May, J. C. (eds) (2004). Freeze-Drying/Lyophilization Of Pharmaceutical \& Biological Products, Second Edition: Revised And Expanded, 2 edition edn, CRC Press, New York.

Rocha-Guzman, N. E., Gallegos-Infante, J. A., GonzalezLaredo, R. F., Harte, F., Medina-Torres, L., Ochoa-Martinez, L. A. and Soto-Garcia, M. (2010). Effect of High-Pressure Homogenization on the Physical and Antioxidant Properties of
Quercus resinosa Infusions Encapsulated by Spray-Drying, Journal of Food Science 75(5): N57-N61.

Shao, J., Li, X., Lu, X., Jiang, C., Hu, Y., Li, Q., You, Y. and Fu, Z. (2009). Enhanced growth inhibition effect of Resveratrol incorporated into biodegradable nanoparticles against glioma cells is mediated by the induction of intracellular reactive oxygen species levels, Colloids and Surfaces B: Biointerfaces 72(1): 40-47.

Singh, M., Hemant, K., Ram, M. and Shivakumar, H. (2010). Microencapsulation: A promising technique for controlled drug delivery, Research in Pharmaceutical Sciences 5(2): 65-77.

Snyder, H. and Lechuga-Ballesteros, D. (2008). Spray Drying: Theory and Pharmaceutical Applications, in L. Augsburger and S. Hoag (eds), Pharmaceutical Dosage Forms - Tablets, CRC Press, pp. 227-260.

Sosa, M., Rodriguez-Rojo, S., Mattea, F., Cismondi, M. and Cocero, M. (2011). Green tea encapsulation by means of high pressure antisolvent coprecipitation, The Journal of Supercritical Fluids 56(3): 304-311.

Sovilj, V., Dokić, P. and Mešinkovska, D. (2000). Investigations of the microencapsulation in the system anionic polyelectrolyte cationic surfactant, Acta Periodica Technologica (Yugoslavia), number 31, Univerzitet u Novom Sadu, Novi Sad (Yugoslavia). Tehnoloski fakultet, pp. 453-549.

Stojanovic, R., Belscak-Cvitanovic, A., Manojlovic, V., Komes, D., Nedovic, V. and Bugarski, B. (2012). Encapsulation of thyme (Thymus serpyllum L.) aqueous extract in calcium alginate beads, Journal of the Science of Food and Agriculture 92(3): 685-696.

Suri, S., Ruan, G., Winter, J. and Schmidt, C. (2013). Microparticles and Nanoparticles, in B. Ratner, A. Hoffman, F. Schoen and J. Lemons (eds), Biomaterials Science, 3rd edn, Elsevier, pp. $360-388$

Swami, H., Kataria, M. K., Bilandi, A., Kour, P. and Bala, S. (2015). Liposome: An art for drug delivery, 5: 9.

Tang, X. C. and Pikal, M. J. (2004). Design of Freeze-Drying Processes for Pharmaceuticals: Practical Advice, Pharmaceutical Research 21(2): 191-200.

Tomšik, A. (2018). Drying and extraction of the wild garlic leaves (Allium ursinum L.) in order to obtain functional products with bioactive potential, PhD thesis, University of Novi Sad, Technological faculty, Serbia.

Zuidam, N. J. and Shimoni, E. (2010). Overview of Microencapsulates for Use in Food Products or Processes and Methods to Make Them, in N. Zuidam and V. Nedovic (eds), Encapsulation Technologies for Active Food Ingredients and Food Processing, Springer New York, New York, NY, pp. 3-29. 